Artículo

\title{
Aporte nutrimental de especies arbóreas fijadoras de nitrógeno en sistemas agroforestales con café
}

\author{
Rubén Garza Lau \\ Ranferi Maldonado Torres ${ }^{\S}$ \\ Ma. Edna Álvarez Sánchez \\ Julio César Buendía Espinoza \\ Maestría en Ciencias en Agroforestería para el Desarrollo Sostenible-Universidad Autónoma Chapingo. \\ Carretera México-Texcoco km 38.5, Chapingo, Texcoco, Estado de México. CP. 56230. \\ ${ }^{\S}$ Autor para correspondencia: ranferimt@yahoo.com.mx.
}

\section{Resumen}

En la región de Huatusco, Veracruz se realizó un experimento para comprobar el efecto que tiene el uso adecuado de fertilizante en la producción de café bajo sombra de especies arbóreas fijadoras de nitrógeno. Para ello, se plantearon cuatro tratamientos en cuatro sistemas agroforestales (caféInga vera, café-Juglans pyriformis, café-Erythrina poeppigiana y café-E. poeppigiana-Grevillea robusta), con tres bloques de 10 plantas cada uno, los cuales consistieron en: a) sin fertilización; b) fertilización al suelo; c) fertilización foliar; d) fertilización al suelo y foliar. El balance nutrimental se evaluó mediante dos análisis foliares realizados antes y después de la fertilización. El rendimiento se evaluó durante los meses de noviembre y diciembre. Con el manejo nutrimental propuesto se elevaron las concentraciones de $\mathrm{N}, \mathrm{P}, \mathrm{K}, \mathrm{Ca}, \mathrm{Mg}$ y $\mathrm{Mn}$, mientras que disminuyeron las de $\mathrm{Fe}, \mathrm{Cu}$ y $\mathrm{Zn}$, acercándose a los niveles óptimos para el cultivo. No se encontraron diferencias significativas $(p \leq 0.05)$ en las concentraciones de nutrientes entre los cuatro tratamientos en los sistemas IV y EG. En EP, hubo diferencia para Ca y Mn, en los tratamientos 2 y 3 . En JP hubo diferencia para $\mathrm{Mg}$ en los tres tratamientos.

Palabras clave: análisis foliar, balance nutrimental, fijación de nitrógeno, rendimiento.

Recibido: enero de 2020

Aceptado: marzo de 2020 


\section{Introducción}

El cultivo de café en México se realiza predominantemente bajo sombra con algunas especies leguminosas que forman asociaciones con bacterias fijadoras de nitrógeno. Dichas bacterias se encuentran en casi todos los ecosistemas de manera libre o en asociación con leguminosas y otras especies, teniendo las segundas una ventaja competitiva al obtener carbohidratos de su hospedero (Saha et al., 2017).

El proceso de nodulación comienza cuando la planta envía señales químicas a través del sistema radicular, las cuales atraen a la bacteria, que se introduce por el pelo radical, provocando la división celular y el rizado de la raíz que se convertirá en nódulo para fijar $\mathrm{N}_{2}$ (Murray, 2011). Para ello, la proteína reductasa provee electrones a la nitrogenasa, la cual reduce el $\mathrm{N}_{2}$ a $\mathrm{NH}_{3}$, cuya síntesis depende tanto del hospedero como de la bacteria.

Mismos que desarrollan mecanismos como la compartimentalización o la síntesis de leghemoglobina para evitar que el oxígeno entre en contacto con la nitrogenasa, pues provoca su inhibición (Juliana, 2004). Dicho proceso implica un gasto considerable de energía para la planta, pues en condiciones ideales, se hidrolizan 16 moléculas de ATP, mientras que en condiciones naturales es menos eficiente.

Los nódulos bacterianos presentan una respuesta positiva a la fertilización química siempre y cuando se tenga un balance nutrimental, ya que la aplicación excesiva de nitrógeno reduce su efectividad hasta inactivarlos (Zheng et al., 2016). En algunos casos, las bacterias nitrificantes pueden suministrar el $\mathrm{N}$ que el cultivo necesita; sin embargo, la inoculación de dichos organismos es indispensable para el proceso de fijación al igual que los elementos Mo, Fe, Ca y Co (Saha et al., 2017; Pedrozo et al., 2018).

Se ha demostrado que en plantaciones de café bajo sombra con especies leguminosas, el rendimiento puede llegar a $3.43 \mathrm{t} \mathrm{ha}^{-1}$ mientras que, en sistemas de café a cielo abierto, el rendimiento se reduce a $1.48 \mathrm{t} \mathrm{ha}^{-1}$ con un máximo de $2 \mathrm{t} \mathrm{ha}^{-1}$ (Peeters et al., 2003; Farfán-Valencia y Mestre-Mestre, 2004; Benítez et al., 2015).

El objetivo del presente estudio fue evaluar la respuesta a la fertilización foliar y al suelo de cuatro plantaciones de café con y sin especies leguminosas, mediante el uso de análisis foliar para proponer un plan de manejo que promueva la fijación biológica de nitrógeno, el reciclaje de nutrientes, mejore el estado nutrimental del cultivo y se obtengan máximos rendimientos.

\section{Materiales y métodos}

El presente estudio se llevó a cabo en el municipio de Huatusco, Veracruz, donde se realizó un experimento en bloques completos al azar (BCA), con cuatro tratamientos de fertilización: 1) testigo; 2) fertilización al suelo; 3) fertilización foliar; 4) fertilización al suelo y foliar. Se eligieron cuatro sistemas agroforestales los cuales fueron: 1) café-Inga vera; 2) café-Juglans pyriformis; 3) café-Erythrina poeppigiana; y 4) café-E. poeppigiana-Grevillea robusta. Para cada tratamiento se eligieron tres bloques de diez plantas, lo que hizo un total de 480 plantas. 
Después de aplicar el fertilizante al cultivo de café, se evaluó el rendimiento de cosecha en el mes de diciembre, se pesó la producción de café cereza por bloque y se secó para conocer el contenido de humedad. Se realizaron dos análisis foliares, uno antes de aplicar los fertilizantes y el segundo cuatro meses después. La muestra foliar se integró colectando el par de hojas ubicadas en la cuarta posición del ápice hacia la base de la rama, en los cuatro puntos cardinales, por cada planta de cada bloque (Lara, 2005).

Las muestras fueron secadas en estufa a $75^{\circ} \mathrm{C}$ por 72 horas y se molieron en un molino de acero inoxidable. Luego se digirieron con $4 \mathrm{ml}$ de mezcla diácida y $2 \mathrm{ml}$ de peróxido de hidrógeno. Después se analizaron los elementos N, P, K, Ca, Mg, Fe, Mn, Zn, Cu y B (Álvarez-Sánchez y Marín-Campos, 2015).

Los nódulos fueron analizados con microscopio electrónico de barrido en busca del bacteroide y se cuantificó el porcentaje de nutrientes dentro del cuadro de la imagen mediante el análisis EDAX (Energy Dispersive X-ray Analysis). Posteriormente, se colectaron nódulos de los tres sistemas con leguminosas para determinar sus concentraciones de nutrientes.

\section{Resultados y discusión}

\section{Análisis foliar previo a la fertilización}

El primer análisis foliar (Cuadro 1) se realizó durante el mes de julio y mostró deficiencias en N, $\mathrm{K}, \mathrm{Ca}, \mathrm{Mg}$ y Zn, de acuerdo con los intervalos propuestos por Osorio (2012). Por su parte, el P, B, Mo y $\mathrm{Mn}$ se ubican en el intervalo de suficiencia, pero el $\mathrm{Fe}$ y $\mathrm{Cu}$ excedieron las concentraciones recomendadas, lo cual es normal en suelos con $\mathrm{pH}$ ácido, donde las concentraciones de $\mathrm{Al}, \mathrm{Fe}, \mathrm{S}$ y Mn en hojas suelen ser elevadas (Bernal-Forero y Almanza-Merchan, 2016).

Cuadro 1. Valores de concentración nutrimental foliar inicial de los sistemas agroforestales por cada tratamiento.

\begin{tabular}{|c|c|c|c|c|c|c|c|c|c|c|c|}
\hline \multirow{2}{*}{ Tratamiento } & $\mathrm{N}$ & $\mathrm{P}$ & $\mathrm{K}$ & $\mathrm{Ca}$ & $\mathrm{Mg}$ & $\mathrm{Fe}$ & $\mathrm{Cu}$ & $\mathrm{Zn}$ & Mn & B & Mo \\
\hline & \multicolumn{5}{|c|}{$(\%)$} & \multicolumn{6}{|c|}{$\left(\mathrm{mg} \mathrm{kg}^{-1}\right)$} \\
\hline $\mathrm{EP}$ & 1.5 & 0.27 & 1.17 & 0.14 & 0.24 & 154.15 & 11.92 & 9.12 & 74.28 & 31 & 0.31 \\
\hline IV & 1.63 & 0.29 & 1.34 & 0.21 & 0.35 & 157.25 & 17.06 & 7.64 & 67.54 & 29 & 0.28 \\
\hline JP & 1.51 & 0.28 & 1.31 & 0.15 & 0.38 & 160.08 & 16.73 & 7.38 & 81.81 & 31.75 & 0.3 \\
\hline EG & 1.60 & 0.26 & 1.39 & 0.16 & 0.27 & 147.43 & 10.18 & 9.22 & 47.98 & 39.25 & 0.29 \\
\hline
\end{tabular}

$\mathrm{EP}=$ sistema café-Erythrina poeppigiana $; \mathrm{IV}=$ sistema café-Inga vera $; \mathrm{JP}=$ sistema café-Juglans pyriformis; $\mathrm{EG}=$ sistema café-Erythrina poeppigiana y Grevillea robusta; $0=$ testigo; $1=$ fertilización al suelo; $2=$ fertilización foliar; $3=$ fertilización al suelo $y$ foliar.

\section{Desviación del óptimo porcentual (DOP)}

A continuación, se muestra el DOP del primer análisis foliar (Cuadro 2), donde se aprecia que, en la mayoría de los sistemas, los nutrimentos deficientes son $\mathrm{Ca}, \mathrm{N}, \mathrm{B}, \mathrm{K}, \mathrm{P}, \mathrm{Mg}$ y $\mathrm{Mn}$, siendo el primero el de mayor escasez, mientras que el Fe fue el único elemento que no tuvo deficiencia en todos los sistemas. 
Cuadro 2. Desviación del óptimo porcentual para el análisis foliar previo a la fertilización.

\begin{tabular}{|c|c|c|c|c|c|c|c|c|c|c|}
\hline & $\mathrm{N}$ & $\mathrm{P}$ & $\mathrm{K}$ & $\mathrm{Ca}$ & $\mathrm{Mg}$ & $\mathrm{B}$ & $\mathrm{Cu}$ & $\mathrm{Fe}$ & $\mathrm{Mn}$ & $\mathrm{Zn}$ \\
\hline & & & $(\%)$ & & & \multicolumn{5}{|c|}{$\left(\mathrm{mg} \mathrm{kg}^{-1}\right)$} \\
\hline & \multicolumn{10}{|c|}{ Diagnóstico nutrimental inicial (EP) } \\
\hline $\mathrm{CN}$ & 1.50 & 0.27 & 1.17 & 0.14 & 0.24 & 31 & 11.92 & 154.15 & 74.28 & 9.12 \\
\hline DOP & -48 & -10 & -43 & -89 & -29 & -39 & 6 & 26 & -5 & -30 \\
\hline \multirow[t]{2}{*}{ ORN } & \multicolumn{10}{|c|}{$\mathrm{Ca}>\mathrm{N}>\mathrm{K}>\mathrm{B}>\mathrm{Zn}>\mathrm{Mg}>\mathrm{P}>\mathrm{Mn}>\mathrm{Cu}>\mathrm{Fe}$} \\
\hline & \multicolumn{10}{|c|}{ Diagnóstico nutrimental inicial (IV) } \\
\hline $\mathrm{CN}$ & 1.63 & 0.29 & 1.34 & 0.21 & 0.35 & 29 & 17.06 & 157.25 & 67.54 & 7.64 \\
\hline DOP & -44 & -4 & -35 & -84 & 2 & -43 & 52 & 29 & -13 & -42 \\
\hline \multirow[t]{2}{*}{ ORN } & \multicolumn{10}{|c|}{$\mathrm{Ca}>\mathrm{N}>\mathrm{B}>\mathrm{Zn}>\mathrm{K}>\mathrm{Mn}>\mathrm{P}>\mathrm{Mg}>\mathrm{Fe}>\mathrm{Cu}$} \\
\hline & \multicolumn{10}{|c|}{ Diagnóstico nutrimental inicial (JP) } \\
\hline $\mathrm{CN}$ & 1.51 & 0.29 & 1.31 & 0.15 & 0.38 & 28 & 15.68 & 160.08 & 81.81 & 15.68 \\
\hline DOP & -48 & -8 & -37 & -88 & 11 & -45 & 40 & 31 & 5 & -44 \\
\hline \multirow[t]{2}{*}{ ORN } & \multicolumn{10}{|c|}{$\mathrm{Ca}>\mathrm{N}>\mathrm{B}>\mathrm{Zn}>\mathrm{K}>\mathrm{P}>\mathrm{Mn}>\mathrm{Mg}>\mathrm{Fe}>\mathrm{Cu}$} \\
\hline & \multicolumn{10}{|c|}{ Diagnóstico nutrimental inicial (EG) } \\
\hline $\mathrm{CN}$ & 1.6 & 0.26 & 1.39 & 0.16 & 0.27 & 39.25 & 10.18 & 147.43 & 47.98 & 9.22 \\
\hline DOP & -45 & -13 & -33 & -88 & -22 & -23 & -9 & 21 & -38 & -30 \\
\hline ORN & & & & & $>N>$ & $\mathrm{K}>\mathrm{Zn}$ & $\mathrm{B}>\mathrm{Mg}$ & $\mathrm{P}>\mathrm{Cu}>\mathrm{F}$ & & \\
\hline
\end{tabular}

$\mathrm{EP}=$ sistema café-Erythrina poeppigiana $; \mathrm{IV}=$ sistema café-Inga vera $; \mathrm{JP}=$ sistema café-Juglans pyriformis $; \mathrm{EG}=$ sistema café-Erythrina poeppigiana y Grevillea robusta; $\mathrm{CN}=$ composición nutrimental; $\mathrm{DOP}=$ desviación del óptimo porcentual; ORN= orden de requerimiento nutrimental.

\section{Índice de balance de Kenworthy (IBK)}

Para realizar el cálculo del índice de balance de Kenworthy se tomaron como referencia los valores reportados por Fonseca et al. (2018), a partir de los cuales se obtuvieron los resultados presentados en el (Cuadro 3), donde se comparan los datos del análisis inicial.

Cuadro 3. Diagnóstico nutrimental utilizando el índice de balance de Kenworthy para el análisis foliar previo a la fertilización.

\begin{tabular}{|c|c|c|c|c|c|c|c|c|c|c|}
\hline & $\mathrm{N}$ & $\mathrm{P}$ & $\mathrm{K}$ & $\mathrm{Ca}$ & $\mathrm{Mg}$ & $\mathrm{B}$ & $\mathrm{Cu}$ & $\mathrm{Fe}$ & $\mathrm{Mn}$ & $\mathrm{Zn}$ \\
\hline & & & $(\%)$ & & & \multicolumn{5}{|c|}{$\left(\mathrm{mg} \mathrm{kg}^{-1}\right)$} \\
\hline & \multicolumn{10}{|c|}{ Diagnóstico nutrimental inicial (EP) } \\
\hline $\mathrm{CN}$ & 1.50 & 0.27 & 1.17 & 0.14 & 0.24 & 31 & 11.92 & 154.15 & 74.28 & 9.12 \\
\hline IBK & 54 & 89 & 60 & 19 & 74 & 75 & 108 & 123 & 97 & 75 \\
\hline $\mathrm{C}$ & $\mathrm{B}$ & $\mathrm{N}$ & $\mathrm{B}$ & MB & $\mathrm{B}$ & $\mathrm{B}$ & $\mathrm{N}$ & $\mathrm{A}$ & $\mathrm{N}$ & $\mathrm{B}$ \\
\hline ORN & & \multicolumn{9}{|c|}{$\mathrm{Ca}>\mathrm{N}>\mathrm{K}>\mathrm{B}>\mathrm{Mg}>\mathrm{Zn}>\mathrm{P}>\mathrm{Mn}>\mathrm{Cu}>\mathrm{Fe}$} \\
\hline
\end{tabular}




\begin{tabular}{|c|c|c|c|c|c|c|c|c|c|c|}
\hline & $\mathrm{N}$ & $\mathrm{P}$ & $\mathrm{K}$ & $\mathrm{Ca}$ & $\mathrm{Mg}$ & $\mathrm{B}$ & $\mathrm{Cu}$ & $\mathrm{Fe}$ & $\mathrm{Mn}$ & $\mathrm{Zn}$ \\
\hline & & & $(\%)$ & & & \multicolumn{5}{|c|}{$\left(\mathrm{mg} \mathrm{kg}^{-1}\right)$} \\
\hline & \multicolumn{10}{|c|}{ Diagnóstico nutrimental inicial (IV) } \\
\hline $\mathrm{CN}$ & 1.63 & 0.29 & 1.34 & 0.21 & 0.35 & 29 & 17.06 & 157.25 & 67.54 & 7.64 \\
\hline IBK & 58 & 95 & 67 & 24 & 102 & 64 & 168 & 125 & 92 & 66 \\
\hline $\mathrm{C}$ & $\mathrm{B}$ & $\mathrm{N}$ & $\mathrm{B}$ & MB & $\mathrm{N}$ & $\mathrm{B}$ & MA & A & $\mathrm{N}$ & $\mathrm{B}$ \\
\hline \multirow[t]{2}{*}{ ORN } & \multicolumn{10}{|c|}{$\mathrm{Ca}>\mathrm{N}>\mathrm{B}>\mathrm{Zn}>\mathrm{K}>\mathrm{Mn}>\mathrm{P}>\mathrm{Mg}>\mathrm{Fe}>\mathrm{Cu}$} \\
\hline & \multicolumn{10}{|c|}{ Diagnóstico nutrimental inicial (JP) } \\
\hline $\mathrm{CN}$ & 1.51 & 0.29 & 1.31 & 0.15 & 0.38 & 28 & 15.68 & 160.08 & 81.81 & 15.68 \\
\hline IBK & 54 & 90 & 66 & 20 & 110 & 62 & 152 & 127 & 107 & 64 \\
\hline $\mathrm{C}$ & $\mathrm{B}$ & $\mathrm{N}$ & $\mathrm{B}$ & MB & $\mathrm{N}$ & $\mathrm{B}$ & MA & A & $\mathrm{N}$ & $\mathrm{B}$ \\
\hline \multirow[t]{2}{*}{ ORN } & \multicolumn{10}{|c|}{$\mathrm{Ca}>\mathrm{N}>\mathrm{B}>\mathrm{Zn}>\mathrm{K}>\mathrm{P}>\mathrm{Mn}>\mathrm{Mg}>\mathrm{Fe}>\mathrm{Cu}$} \\
\hline & \multicolumn{10}{|c|}{ Diagnóstico nutrimental inicial (EG) } \\
\hline $\mathrm{CN}$ & 1.60 & 0.26 & 1.39 & 0.16 & 0.27 & 39.25 & 10.18 & 147.43 & 47.98 & 9.22 \\
\hline IBK & 57 & 85 & 70 & 20 & 81 & 81 & 93 & 118 & 78 & 76 \\
\hline $\mathrm{C}$ & $\mathrm{B}$ & $\mathrm{N}$ & $\mathrm{B}$ & MB & $\mathrm{B}$ & $\mathrm{B}$ & $\mathrm{N}$ & A & $\mathrm{B}$ & $\mathrm{B}$ \\
\hline ORN & & & & & $>N>$ & $\mathrm{Zn}>\mathrm{Mn}$ & $\mathrm{Mg}>\mathrm{B}$ & $\mathrm{P}>\mathrm{Cu}>\mathrm{F}$ & & \\
\hline
\end{tabular}

$\mathrm{EP}=$ sistema café-Erythrina poeppigiana $; \mathrm{IV}=$ sistema café-Inga vera $; \mathrm{JP}=$ sistema café-Juglans pyriformis; $\mathrm{EG}=$ sistema café-Erythrina poeppigiana y Grevillea robusta; $\mathrm{CN}=$ composición nutrimental; $\mathrm{IBK}=$ índice de balance de Kenworthy; $\mathrm{C}=$ condición; $\mathrm{MB}=$ muy baja; $\mathrm{B}=$ baja; $\mathrm{N}=$ normal; $\mathrm{A}=$ alta; $\mathrm{MA}=$ muy alta; $\mathrm{ORN}=$ orden de requerimiento nutrimental.

Ambas metodologías de interpretación de análisis foliar coinciden en que los elementos más deficientes en el cultivo son $\mathrm{Ca}, \mathrm{N}, \mathrm{B}, \mathrm{K}$ y Mg, mientras que Fe y $\mathrm{Cu}$ llegan a niveles de exceso, lo que podría provocar problemas en el cultivo por toxicidad (López-García et al., 2016).

\section{Análisis foliar posterior a la fertilización}

El segundo análisis foliar se llevó a cabo durante el mes de noviembre, en él se refleja una diferencia en la concentración de $\mathrm{N}$ en los sistemas IV, EG y EP superior para los tratamientos 2 y 3, demostrando la efectividad de la fertilización foliar y al suelo. En el Cuadro 4 se muestra el resultado del análisis foliar.

Cuadro 4. Estado nutrimental de los sistemas por tratamiento después de aplicar los productos.

\begin{tabular}{|c|c|c|c|c|c|c|c|c|c|c|}
\hline \multirow{2}{*}{ Tratamiento } & $\mathrm{N}$ & $\mathrm{P}$ & $\mathrm{K}$ & $\mathrm{Ca}$ & $\mathrm{Mg}$ & $\mathrm{Fe}$ & $\mathrm{Zn}$ & $\mathrm{Mn}$ & $\mathrm{Cu}$ & $\mathrm{B}$ \\
\hline & \multicolumn{5}{|c|}{$(\%)$} & \multicolumn{5}{|c|}{$\left(\mathrm{mg} \mathrm{kg}^{-1}\right)$} \\
\hline EP & 1.89 & 0.21 & 2.89 & 0.54 & 0.27 & 61.9 & 5.17 & 84.74 & 7.33 & 129.53 \\
\hline IV & 1.9 & 0.24 & 2.85 & 0.95 & 0.36 & 146.43 & 7.7 & 212.56 & 7.69 & 80.48 \\
\hline JP & 2.05 & 0.18 & 3.06 & 0.82 & 0.33 & 69.47 & 3.73 & 201.39 & 8.41 & 117.14 \\
\hline EG & 1.99 & 0.23 & 2.74 & 0.8 & 0.26 & 69.77 & 5.41 & 115.38 & 8.63 & 119.05 \\
\hline
\end{tabular}

$\mathrm{EP}=$ sistema café-Erythrina poeppigiana $; \mathrm{IV}=$ sistema café-Inga vera $; \mathrm{JP}=$ sistema café-Juglans pyriformis $; \mathrm{EG}=$ sistema café-Erythrina poeppigiana y Grevillea robusta. 
El P y K tuvieron una ligera respuesta a los tratamientos, no obstante, en los sistemas EP y JP se puede notar un pequeño incremento en la concentración de dichos nutrientes. Por otro lado, Ca y Mg tuvieron respuesta positiva a los tratamientos 2 y 3, con excepción del sistema EG, que tuvo un valor bajo.

En el caso de los microelementos, $\mathrm{Fe}, \mathrm{Zn}, \mathrm{Mn}$ y $\mathrm{Cu}$ mostraron una respuesta positiva a la fertilización foliar, pero en el tratamiento 3 los valores disminuyeron, sin embargo, de acuerdo con lo reportado por Fonseca et al. (2018), los valores alcanzan niveles que pueden provocar toxicidad en el cultivo, por lo que, al disminuir su concentración en las plantas, se obtiene un mejor balance nutrimental.

En la Figura 1 y Figura 2 se observa que tanto K como B tuvieron un incremento considerable, mientras que las concentraciones de $\mathrm{P}, \mathrm{Fe}, \mathrm{Zn}$ y $\mathrm{Cu}$ disminuyeron ligeramente, lo cual representa un beneficio para el cultivo, pues Fe superaba las concentraciones recomendadas en la literatura (Osorio, 2012). De igual manera, se observa un incremento en las concentraciones de $\mathrm{Ca}$ y $\mathrm{Mg}$, elementos cuya disponibilidad es limitada en suelos con pH ácido.

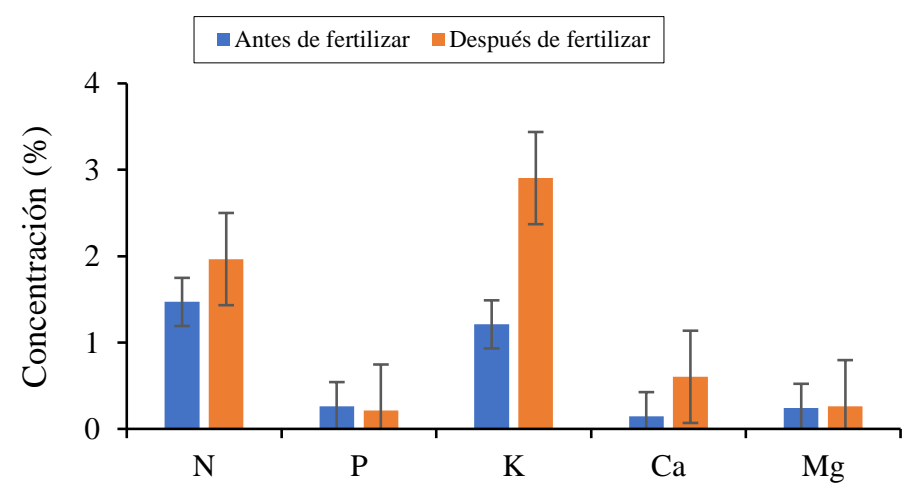

Figura 1. Concentraciones promedio de nutrientes en el sistema EP antes y después de fertilizar.

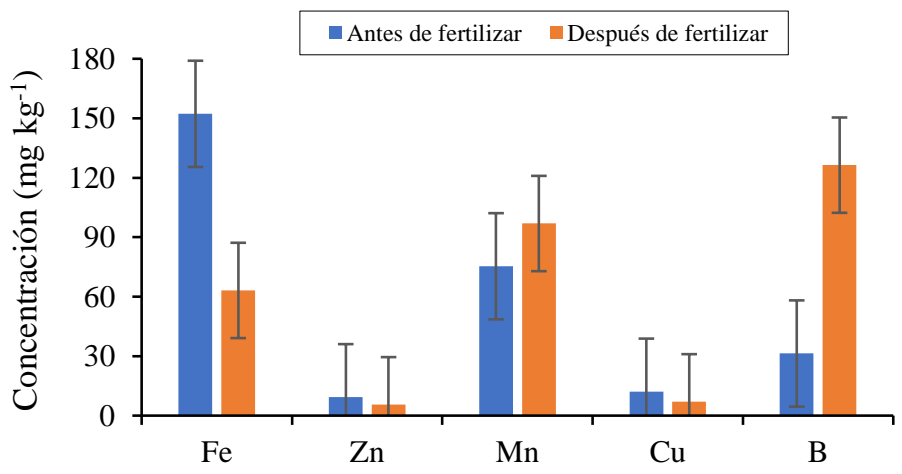

Figura 2. Concentraciones promedio de micronutrientes en el sistema EP antes y después de fertilizar.

En la Figura 3 y Figura 4 se observa que las concentraciones de K, Ca y Mn presentaron una respuesta positiva a la fertilización, pues se incrementaron considerablemente, mientras que $\mathrm{N}, \mathrm{Fe}$, $\mathrm{P}, \mathrm{Mg}, \mathrm{Zn}$ y $\mathrm{Cu}$ no modificaron sus concentraciones de manera considerable, como refiere Rodríguez et al. (2014). 


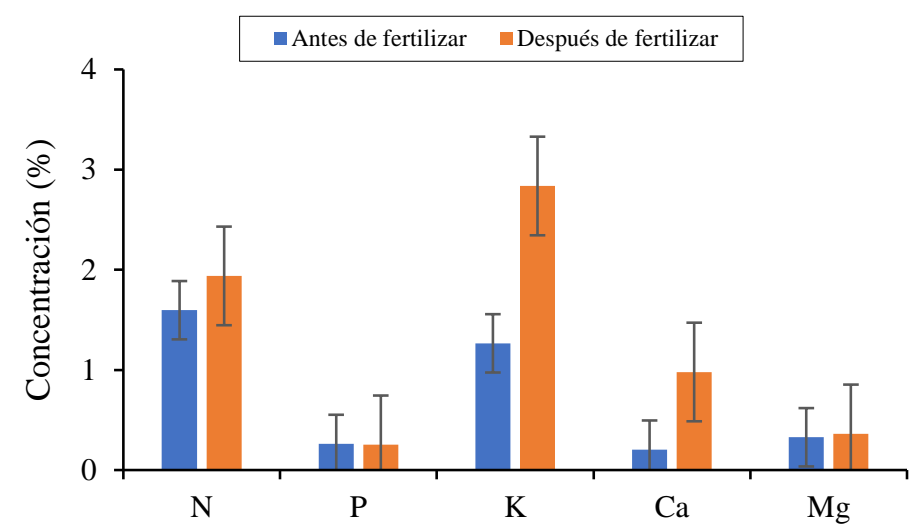

Figura 3. Concentraciones de macronutrientes en el sistema IV antes y después de fertilizar.

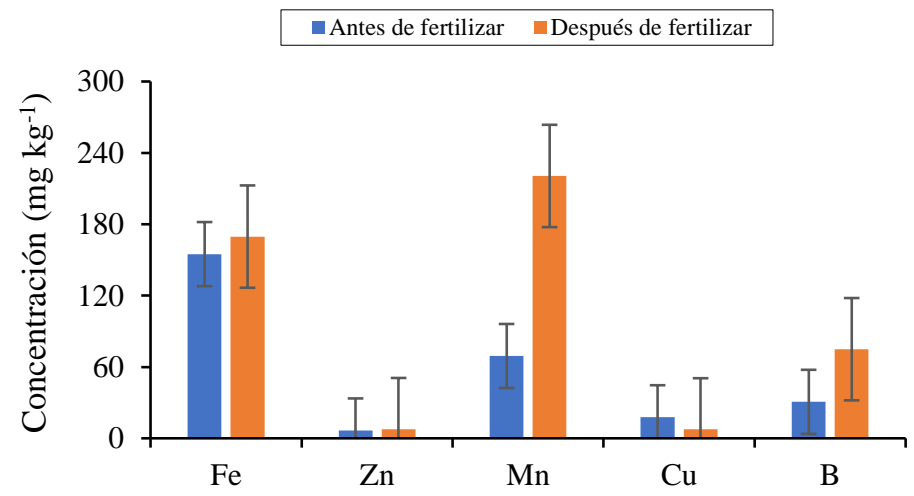

Figura 4. Concentraciones de micronutrientes en el sistema IV antes y después de fertilizar.

En el sistema JP (Figura 5 y Figura 6) los elementos K, Mn y B tuvieron un marcado incremento en respuesta a la fertilización. El Fe redujo su concentración de toxicidad a niveles normales, mientras que los demás elementos no tuvieron una respuesta tan marcada.

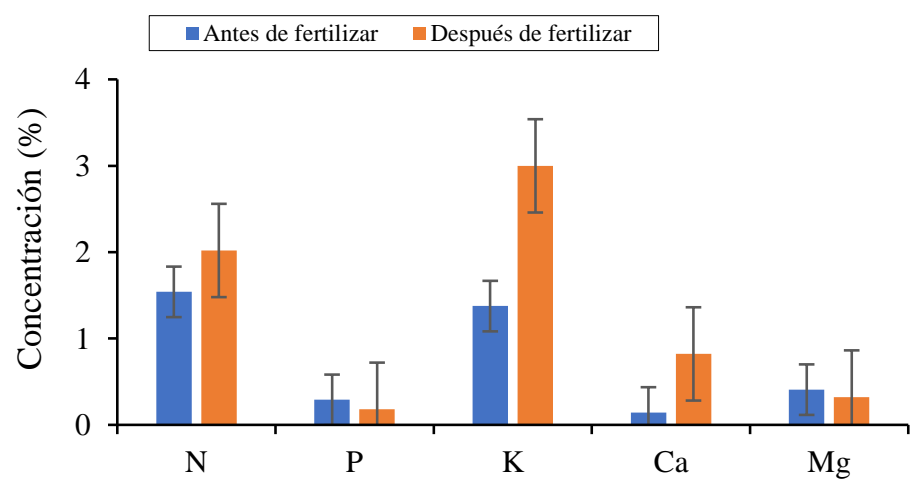

Figura 5. Concentraciones de macronutrientes en el sistema JP antes y después de fertilizar. 


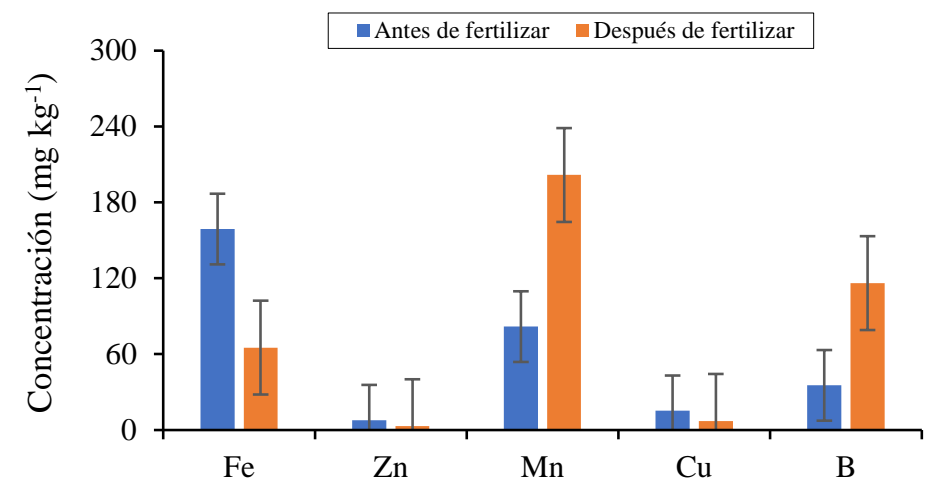

Figura 6. Concentraciones de micronutrientes en el sistema JP antes y después de fertilizar.

En el sistema EG (Figura 7 y Figura 8) se elevaron las concentraciones de nutrientes que, previo a la fertilización, se encontraban en condiciones de deficiencia (K, Ca, Mn y B), mientras que el Fe se redujo de manera considerable. De igual manera, el $\mathrm{P}$ se redujo, debido a que el cultivo presentaba concentraciones muy elevadas del mismo, así lo demuestran investigaciones como las realizadas por Wintgens (2004a).

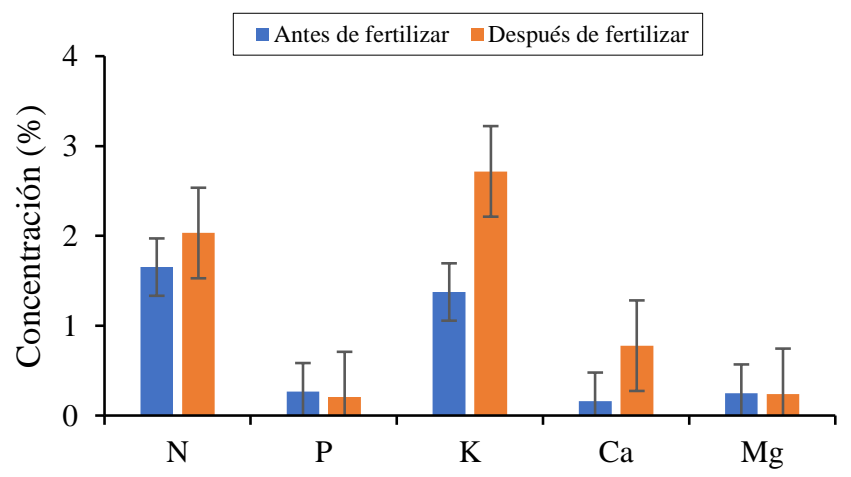

Figura 7. Concentraciones de macronutrientes en el sistema EG antes y después de fertilizar.

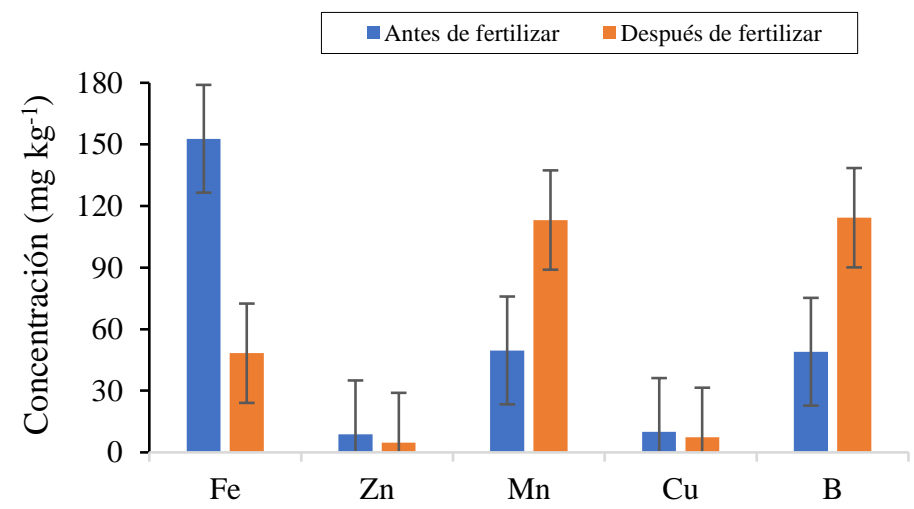

Figura 8. Concentraciones de micronutrientes en el sistema EG antes y después de fertilizar. 


\section{Desviación del óptimo porcentual (DOP)}

En el análisis foliar posterior a la aplicación de fertilizantes, el DOP muestra un importante incremento en los niveles de $\mathrm{Ca}$, B y $\mathrm{K}$, se observa en el Cuadro 5 elevando los primeros dos a niveles aceptables para la planta, mientras que $\mathrm{Zn}, \mathrm{Fe}, \mathrm{N}$ y $\mathrm{Cu}$ permanecen en concentraciones por debajo del óptimo propuesto por Fonseca et al. (2018); INIFAP (2013).

Cuadro 5. Desviación del óptimo porcentual para el análisis foliar posterior a la fertilización.

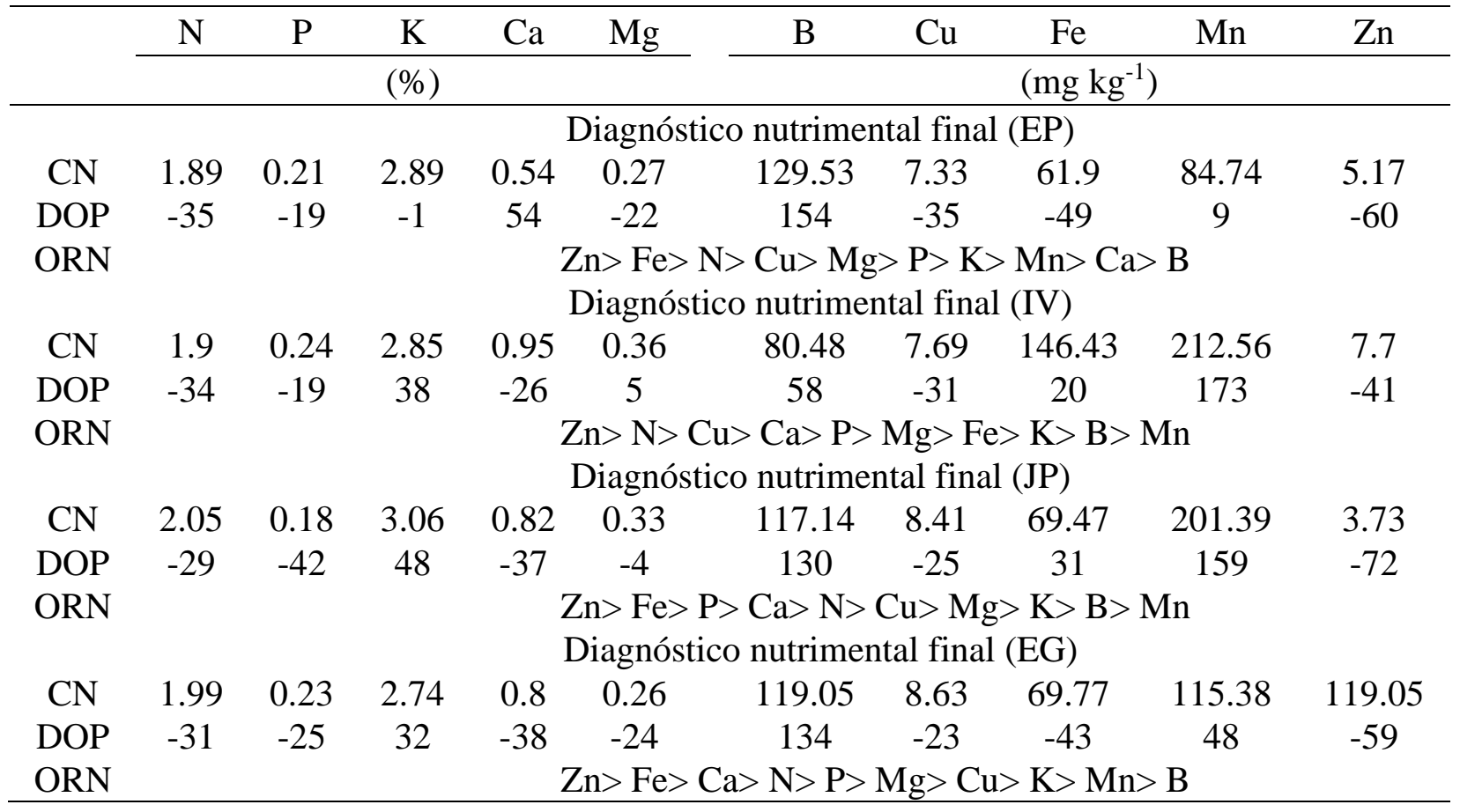

$\mathrm{EP}=$ sistema café-Erythrina poeppigiana $; \mathrm{IV}=$ sistema café-Inga vera $; \mathrm{JP}=$ sistema café-Juglans pyriformis $; \mathrm{EG}=$ sistema café-Erythrina poeppigiana y Grevillea robusta; $\mathrm{CN}=$ composición nutrimental; $\mathrm{DOP}=$ desviación del óptimo porcentual; $\mathrm{ORN}=$ orden de requerimiento nutrimental.

\section{Índice de balance de Kenworthy (IBK)}

El IBK muestra un incremento en las concentraciones de Ca en IV, EG y JP, mientras que N, K, B y Mn incrementaron en todos los sistemas, llegando los tres últimos a niveles excedentes. Por otra parte, $\mathrm{Mg}$ se mantuvo en concentraciones normales y $\mathrm{Fe}, \mathrm{Cu}, \mathrm{P}$ y $\mathrm{Zn}$ disminuyeron (Cuadro 6).

Cuadro 6. Diagnóstico nutrimental utilizando el índice de balance de Kenworthy para el análisis foliar posterior a la fertilización.

\begin{tabular}{|c|c|c|c|c|c|c|c|c|c|c|}
\hline & $\mathrm{N}$ & $\mathrm{P}$ & $\mathrm{K}$ & $\mathrm{Ca}$ & $\mathrm{Mg}$ & B & $\mathrm{Cu}$ & $\mathrm{Fe}$ & $\mathrm{Mn}$ & $\mathrm{Zn}$ \\
\hline \multicolumn{6}{|c|}{$(\%)$} & \multicolumn{5}{|c|}{$\left(\mathrm{mg} \mathrm{kg}^{-1}\right)$} \\
\hline & \multicolumn{10}{|c|}{ Diagnóstico nutrimental final (EP) } \\
\hline $\mathrm{CN}$ & 1.89 & 0.21 & 2.88 & 0.54 & 0.27 & 129.5 & 7.33 & 61.9 & 84.74 & 5.17 \\
\hline IBK & 67 & 66 & 137 & 47 & 81 & 229 & 55 & 58 & 105 & 50 \\
\hline $\mathrm{C}$ & B & B & A & MB & B & MA & B & B & $\mathrm{N}$ & B \\
\hline ORN & & & & & $\mathrm{Zn}>$ & $\mathrm{Fe}>\mathrm{P}>$ & $\mathrm{Mg}>$ & $\mathrm{In}>\mathrm{K}$ & & \\
\hline
\end{tabular}




\begin{tabular}{|c|c|c|c|c|c|c|c|c|c|c|}
\hline & $\mathrm{N}$ & $\mathrm{P}$ & $\mathrm{K}$ & $\mathrm{Ca}$ & $\mathrm{Mg}$ & B & $\mathrm{Cu}$ & $\mathrm{Fe}$ & $\mathrm{Mn}$ & $\mathrm{Zn}$ \\
\hline \multicolumn{6}{|c|}{$(\%)$} & \multicolumn{5}{|c|}{$\left(\mathrm{mg} \mathrm{kg}^{-1}\right)$} \\
\hline & \multicolumn{10}{|c|}{ Diagnóstico nutrimental final (IV) } \\
\hline $\mathrm{CN}$ & 1.9 & 0.24 & 2.85 & 0.95 & 0.36 & 80.48 & 7.69 & 146.4 & 212.6 & 7.7 \\
\hline IBK & 67 & 78 & 135 & 76 & 105 & 148 & 59 & 117 & 199 & 66 \\
\hline $\mathrm{C}$ & $\mathrm{B}$ & $\mathrm{B}$ & A & $\mathrm{B}$ & $\mathrm{N}$ & A & B & $\mathrm{N}$ & MA & B \\
\hline ORN & \multicolumn{10}{|c|}{$\begin{array}{l}\mathrm{Cu}>\mathrm{Zn}>\mathrm{N}>\mathrm{Ca}>\mathrm{P}>\mathrm{Mg}>\mathrm{Fe}>\mathrm{K}>\mathrm{B}>\mathrm{Mn} \\
\text { Diagnóstico nutrimental final }(\mathrm{JP})\end{array}$} \\
\hline $\mathrm{CN}$ & 2.05 & 0.18 & 3.06 & 0.82 & 0.33 & 117.14 & 8.41 & 69.47 & 201.39 & 3.73 \\
\hline IBK & 72 & 52 & 144 & 67 & 96 & 209 & 67 & 63 & 326 & 41 \\
\hline $\mathrm{C}$ & B & B & A & B & $\mathrm{N}$ & MA & B & $\mathrm{B}$ & MA & MB \\
\hline ORN & \multicolumn{10}{|c|}{$\begin{array}{l}\mathrm{Zn}>\mathrm{P}>\mathrm{Fe}>\mathrm{Cu}>\mathrm{Ca}>\mathrm{N}>\mathrm{Mg}>\mathrm{K}>\mathrm{B}>\mathrm{Mn} \\
\text { Diagnóstico nutrimental final }(\mathrm{EG})\end{array}$} \\
\hline $\mathrm{CN}$ & 1.99 & 0.23 & 2.74 & 0.8 & 0.26 & 119.05 & 8.63 & 69.77 & 115.38 & 5.41 \\
\hline IBK & 70 & 71 & 130 & 65 & 79 & 212 & 84 & 63 & 128 & 52 \\
\hline $\mathrm{C}$ & $\mathrm{B}$ & $\mathrm{B}$ & $\mathrm{A}$ & $\mathrm{B}$ & B & A & $\mathrm{N}$ & B & $\mathrm{A}$ & B \\
\hline ORN & \multicolumn{10}{|c|}{$\mathrm{Zn}>\mathrm{Fe}>\mathrm{Ca}>\mathrm{N}>\mathrm{P}>\mathrm{Mg}>\mathrm{Cu}>\mathrm{Mn}>\mathrm{K}>\mathrm{B}$} \\
\hline
\end{tabular}

$\mathrm{EP}=$ sistema café-Erythrina poeppigiana $; \mathrm{IV}=$ sistema café-Inga vera $; \mathrm{JP}=$ sistema café-Juglans pyriformis; $\mathrm{EG}=$ sistema café-Erythrina poeppigiana y Grevillea robusta; $\mathrm{CN}=$ composición nutrimental; $\mathrm{IBK}=$ índice de Balance de Kenworthy; $\mathrm{C}=$ condición; $\mathrm{MB}=$ muy baja; $\mathrm{B}=$ baja; $\mathrm{N}=$ normal; $\mathrm{A}=$ alta; $\mathrm{MA}=$ muy alta; $\mathrm{ORN}=$ orden de requerimiento nutrimental.

Ambas metodologías de interpretación de análisis muestran que los niveles de N, P, K, Ca, Mg y $\mathrm{Mn}$ se elevaron, mientras las concentraciones de $\mathrm{Fe}, \mathrm{Cu}$ y $\mathrm{Zn}$ disminuyeron a niveles aceptables, demostrando que el manejo propuesto mejora el balance nutrimental del cultivo de café (Figura 9).

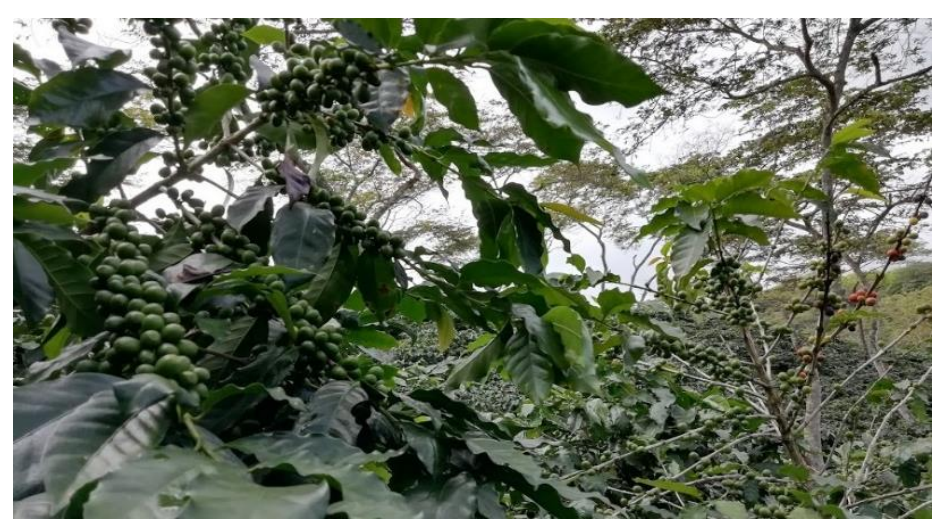

Figura 9. Comparación de plantas de café, a la izquierda el tratamiento 3 en el sistema JP, a la derecha una planta bajo el manejo del productor.

\section{Análisis estadístico}

Los datos obtenidos a partir de los análisis foliares se procesaron con ayuda del software R (ver. 3.6.1) para obtener el Anova (resumido mediante el $p$-value) y comparación de medias con Tukey y Duncan. En el Cuadro 7 se muestran los resultados del ANOVA por sistema agroforestal para 
cada elemento, representado por el $p$-value. En los casos donde se rechaza la Ho, se muestra también la comparación de medias con prueba de Tukey y de Duncan, para señalar el tratamiento que tuvo una diferencia significativa. Los sistemas IV y EG no demostraron diferencia significativa a pesar de haber cambiado las concentraciones nutrimentales después de la fertilización (Pérez y Argueta, 2011).

Cuadro 7. Análisis estadístico de los tratamientos para el sistema EP por elemento $(p \leq 0.05)$.

\begin{tabular}{cccc}
\hline Sistema & Elemento & P-value & Decisión \\
\hline EP & $\mathrm{N}$ & 0.1868 & Ho \\
& $\mathrm{P}$ & 0.7322 & Ho \\
$\mathrm{K}$ & 0.2755 & Ho \\
$\mathrm{Ca}$ & 0.0105 & $\mathrm{R} \mathrm{Ho}$ \\
$\mathrm{Mg}$ & 0.1954 & Ho \\
$\mathrm{Fe}$ & 0.5635 & Ho \\
$\mathrm{Cu}$ & 0.4011 & Ho \\
$\mathrm{Zn}$ & 0.4175 & Ho \\
$\mathrm{Mn}$ & 0.0021 & R Ho \\
& $\mathrm{B}$ & 0.3435 & Ho \\
\hline
\end{tabular}

$\mathrm{EP}=$ sistema de Erythrina poeppigiana $\mathrm{Ho}=$ se acepta la hipótesis nula; $\mathrm{R} \mathrm{Ho}=$ se rechaza la hipótesis nula, se acepta la alternativa.

\section{Nodulación}

En los sistemas EP, IV, se observó nodulación, pero al realizar cortes a los nódulos, se pudo apreciar que estos carecían de leghemoglobina (color rosa a rojo) o se encontraba en concentraciones muy bajas. En el sistema EG se observó nodulación muy escasa, posiblemente debido a la edad del arbolado, $\mathrm{pH}$ y carencia de activadores para la nodulación (Co, Mo, $\mathrm{Fe}$ ).

En los nódulos de EP, la bacteria pudo reconocerse fácilmente en imágenes de microscopio electrónico de barrido (Figura 10), mientras que en IV y EG fue más complicado (Figura 11), quizá debido a la baja presencia de leghemoglobina. Sin embargo, la cuantificación del porcentaje de cada nutriente mediante la respuesta espectral demuestra altos niveles de Mo.

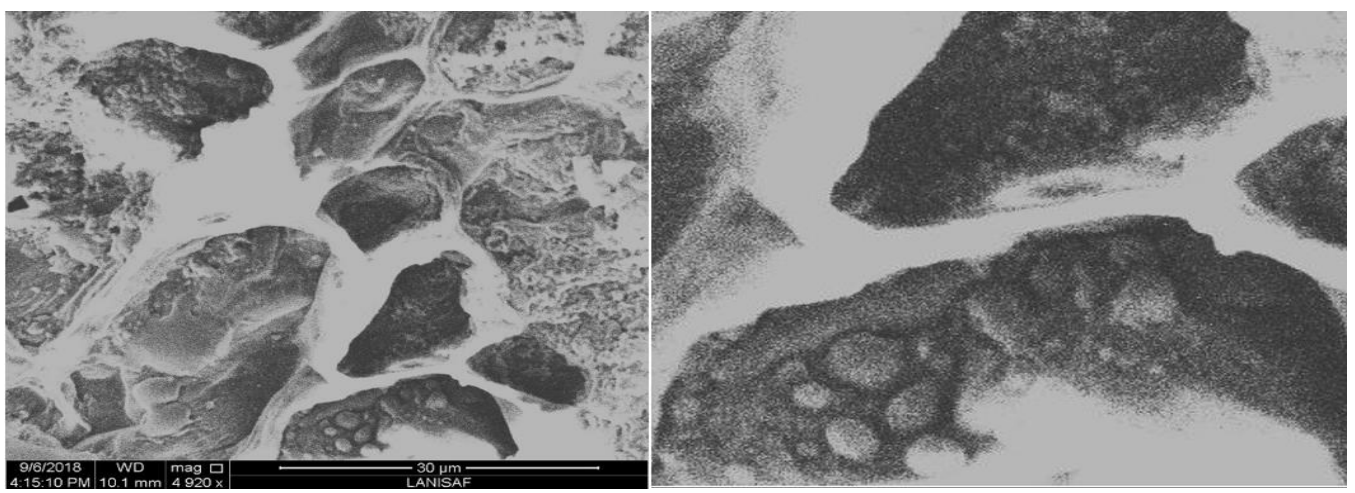

Figura 10. Imagen de microscopio electrónico de barrido en un nódulo de EP. A la izquierda, la imagen completa, a la derecha, un acercamiento a la zona donde se identificó el bacteroide. 


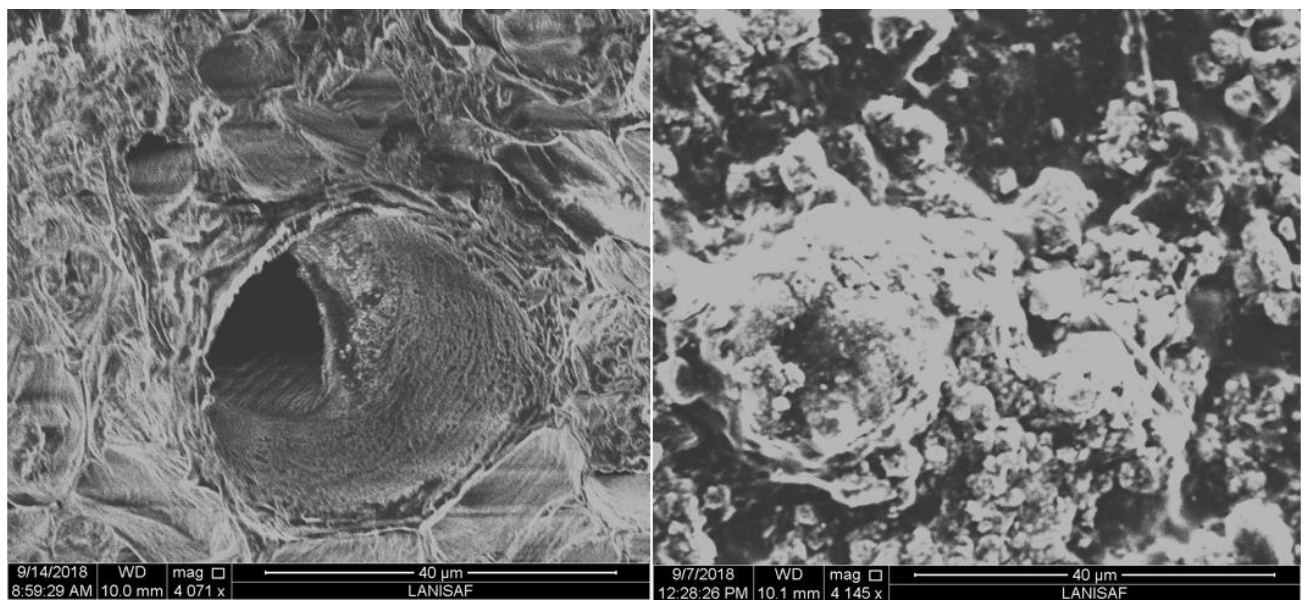

Figura 11. Imágenes de microscopio electrónico de barrido en nódulos de IV (izquierda) y EG (derecha).

En los tres nódulos analizados (Cuadro 8), ya que dicho elemento promueve la inserción de electrones en el $\mathrm{N}_{2}$ y el movimiento de este elemento dentro de la planta, elevando el rendimiento de cultivos como el frijol (Olivé, 2009; Ocaña-Reyes, 2016; Pérez y Ruiz, 2017).

Cuadro 8. Concentraciones de nitrógeno, hierro, cobalto y molibdeno en las imágenes capturadas por el microscopio electrónico de barrido.

\begin{tabular}{cccc}
\hline Elemento/sistema & EP & IV & EG \\
\hline N (\%) en peso & 3.52 & 22.52 & 6.86 \\
Fe (\%) en peso & 3.23 & 0.55 & 2.89 \\
Co (\%) en peso & 1.27 & 0.78 & 2.11 \\
Mo (\%) en peso & 13.9 & 17.77 & 9.64 \\
\hline
\end{tabular}

En los nódulos de IV se observaron estructuras parecidas a canales que se aprecian en EP, lo cual indica que los nódulos se formaron correctamente; sin embargo, no se observaron bacteroides dentro de dichas estructuras. Por otro lado, en EG no se observa desarrollo estructural ni bacteroide, debido a la inactividad del nódulo.

En el análisis cuantitativo de las imágenes, se detectaron altas concentraciones de nitrógeno en los nódulos de ambas especies, demostrando que las raíces están absorbiendo dicho elemento; a pesar, de la escasa presencia de leghemoglobina. De igual manera, el Fe y Co, que son los elementos activadores de la FBN se encuentran en concentraciones superiores a las determinadas mediante el análisis de suelo, a pesar de la presencia de antagonismos con $\mathrm{el} \mathrm{Al}^{3+}$.

\section{Conclusiones}

No se observó una nodulación abundante en las raíces de los árboles, pero sí en aquellas que están en contacto con el agua de escorrentía en la orilla de la parcela, junto al corte del camino en el sistema EP. Esto puede deberse a que la disponibilidad de nutrientes en esta zona es mayor, ya que 
el agua arrastra cationes como $\mathrm{Ca}^{++} \mathrm{y} \mathrm{Mg}^{++}$. Para solucionar dicho problema, es necesario que el productor continúe fertilizando con el esquema propuesto, para mejorar la producción y promover el reciclaje de nutrientes esenciales para los nódulos. Al comparar ambos análisis foliares, las concentraciones de $\mathrm{N}, \mathrm{P}, \mathrm{K}, \mathrm{Ca}, \mathrm{Mg}$ y $\mathrm{Mn}$ se elevaron hasta llegar a niveles dentro del rango normal, mientras que las concentraciones de $\mathrm{Fe}, \mathrm{Cu}$ y $\mathrm{Zn}$ disminuyeron, acercándose al rango óptimo para el cultivo.

No se encontró diferencia estadísticamente significativa en los sistemas IV y EG, mientras que en EP hubo diferencia en las concentraciones de $\mathrm{Ca}$ y $\mathrm{Mn}$, elementos cuya asimilación es limitada en suelos ácidos. En el caso del $\mathrm{Ca}$, los tratamientos 2 y 3 tuvieron una respuesta positiva y estadísticamente diferente al tratamiento 1, en la concentración de Mn, el tratamiento 3 tuvo una marcada respuesta positiva con respecto a los otros tratamientos. En JP se encontró diferencia en las concentraciones de $\mathrm{Mg}$, siendo el tratamiento 1 aquel que presentó concentraciones más bajas con respecto a los otros tratamientos.

La producción de fruto se vio beneficiada por diferentes tratamientos en cada sistema, mientras que en EP y EG se obtuvieron rendimientos elevados con el tratamiento 3, en IV fue el tratamiento 2 el que produjo el mismo efecto y JP se vio beneficiado por el tratamiento 1, lo cual confirma la necesidad de analizar cada sistema de manera independiente para ofrecer al productor esquemas de fertilización específicos para cada condición edáfica que permita obtener máximos rendimientos.

\section{Literatura citada}

Álvarez-Sánchez, M. E. y Marín-Campos, A. 2015. Manual de procedimientos analíticos de suelo y planta del Laboratorio de Química, Departamento de Suelos. Universidad Autónoma Chapingo (UACH). Chapingo, Estado de México. 3-78 pp.

Benítez-García, E.; Jaramillo-Villanueva, J. L.; Escobedo-Garrido, S. y Mora-Flores, S. 2015. Caracterización de la producción y del comercio de café en el municipio de Cuetzalan, Puebla. Agric. Soc. Des. 12(2):181-198.

Bernal F., A. A.; Forero U., F. E., y Almanza-Merchán, P. J. 2016. Contenido de azufre, aluminio, hierro y manganeso foliar en especies vegetales cultivadas en suelo sulfatado ácido. Revista Colombiana de Ciencias Hortícolas, 2(9):279. https://doi.org/10.17584/rcch.2015 v9i2.4184.

Farfán-Valencia, F. y Mestre-Mestre, A. 2004. Respuesta del café cultivado en un sistema agroforestal a la aplicación de fertilizantes. 55(2):161-174.

Fonseca, A.; Lima, J. and Silva, S. 2018. Spacial variability of balanced indexes of Kenworthy (BIK) for macro and micronutrients on the coffee Canephora. J. Exp. Agric. Inter. 23(1):110. https://doi.org/10.9734/jeai/2018/4121.

INIFAP. 2013. Instituto Nacional de Investigaciones Forestales, Agrícolas y Pecuarias. Cafeticultura en la Zona Centro de Veracruz, Diagnóstico, productividad y servicios ambientales. Centro de investigación regional Golfo Centro, Teocelo, Veracruz. 36 p.

Juliana, M. F. 2004. Fijación biológica de nitrógeno. UDO Agrícola. 4(1):1-20.

Lara, E. L. D. 2005. Efectos de la altitud, sombra, producción y fertilización sobre la calidad del café (Coffea arábica L. var. Caturra) producido en sistemas agroforestales de la zona cafetalera norcentral de Nicaragua. Tesis de Maestría, CATIE. Turrialba, Costa Rica. 92 p. 
López-García, F. J.; Escamilla-Prado, E.; Zamarripa-Colmenero, A. y Cruz- Castillo, J. G. 2016. Producción y calidad en variedades de café (Coffea arabica L.) en Veracruz, México. Rev. Fitotec. Mex. 3(39):297-304.

Murray, J. D. 2011. Invasion by invitation: rhizobial infection in legumes. The American Phytopathological Society. 24(6):631-639.

Ocaña-Reyes, J. A. 2016. Aplicación de molibdeno y cobalto en frijol (Phaseolus vulgaris L.) con dos sistemas de fertilización bajo cero labranza. Universidad Nacional Agraria la Molina.

Olivé, L. 2009. Pluralismo epistemológico. Consejo Latinoamericano de Ciencias Sociales. La Paz, Bolivia. $302 \mathrm{p}$.

Osorio, N. W. 2012. El análisis foliar: una poderosa herramienta para diagnosticar el estado nutricional de los cultivos, pasturas y plantaciones. Manejo Integral del Suelo y Nutrición Vegetal. 3(1):1-4.

Pedrozo, A.; De Oliveira, N. J. G. and Alberton, O. 2018. Biological nitrogen fixation and agronomic features of soybean (Glycine max (L.) Merr.) crop under different doses of inoculant. Acta Agronomica. 67(2):297-302. https://doi.org/10.15446/acag.v67n2.56375.

Peeters, L. Y. K.; Soto-Pinto, L.; Perales, H.; Montoya, G. and Ishiki, M. 2003. Coffee production, timber, and firewood in traditional and Inga-shaded plantations in Southern Mexico. Agric. Ecosys. Environ. 95(2-3):481-493.

Pérez, F. Y. y Ruiz, E. F. 2017. Construyendo autogestión en la producción y conservación del café de calidad en Teocelo, Veracruz. Universidad Autónoma Chapingo, Primera ed., Chapingo, Texcoco, Estado de México: Centro Regional Universitario del Anáhuac. 61 p.

Pérez, R. M. L. y Argueta, V. A. 2011. Saberes indígenas y diálogo intercultural. Rev. Cultura y Representaciones Sociales. 10(5):31-56.

Rodríguez, B. E.; Suarez, S. J. C. y Duran, B. E. H. 2014. Efecto de las condiciones de cultivo, las características químicas del suelo y el manejo de grano en los atributos sensoriales de café (Coffea arabica L.) en taza. Acta Agron. 4(64):342-348.

Saha, B.; Saha, S.; Das, A.; Bhattacharyya, P. K.; Basak, N.; Sinha, A. K. and Poddar, P. 2017. Agriculturally important microbes for sustainable agriculture. Meena, V. S.; Mishra, P. K.; Bisht, J. K. and Pattanayak, A. (Eds.). In: agriculturally important microbes for sustainable agriculture $1^{\text {st }}$ (Ed.). Singapore. https://doi.org/10.1007/978-981-10-5343-6_4.

Wintgens, J. N. 2004a. Factor influencing the quality of Green coffee. In: coffee: growing, processing, sustainable production: a guidebook for growers, processors, traders, and researchers. Wintgens, J. N. (Ed.). Wiley-VHC. Weinhein, Germany. 789-809 pp.

Zheng, M.; Chen, H.; Li, D.; Zhu, X.; Zhang, W.; Fu, S. and Mo, J. 2016. Biological nitrogen fixation and its response to nitrogen input in two mature tropical plantations with and without legume trees. Biol. Fertility Soils. 52(5):665-674. https://doi.org/10.1007/s00374016-1109-5. 